\title{
Case Control Studies in Evaluating Prostate Cancer Screening: An Overview
}

\author{
Suzie J. Otto ${ }^{a, *}$, Monique J. Roobol ${ }^{b}$ \\ a Department of Public Health, Erasmus MC, P.O. Box 2040, 3000 CA Rotterdam, Netherlands \\ ${ }^{\mathrm{b}}$ Department of Urology, Erasmus MC, P.O. Box 2040, 3000 CA Rotterdam, Netherlands
}

\section{Article info}

Keywords:

Prostate cancer

Case-control study

Screening

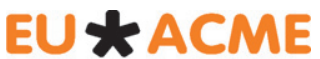

www.eu-acme.org/

europeanurology

\begin{abstract}
Objectives: Ongoing randomized controlled screening trials for prostate cancer have not shown a beneficial effect on prostate cancer mortality reduction yet. A large number of observational (non-randomized) studies on prostate cancer screening have been published with contradictory outcome. This paper reviews the current case-control studies.

Methods: Seven case-control studies of screening for prostate cancer were identified in a PubMed search, published from 1991 onwards, all conducted in North America. The screening test was either digital rectal examination (DRE) alone or in combination with PSA.

Results: One DRE case-control study, found a significant preventive effect, whereas two others showed no effect of DRE screening on prostate cancer mortality nor on the occurrence of metastatic disease. Conflicting results were also observed in the studies assessing the effect of PSA/DRE. Only one study showed a significant $27 \%$ mortality reduction in the White male cohort, but found no effects in Blacks. The most recent study showed that screening with PSA/DRE was not protective in reducing prostate cancer mortality.

Conclusions: Our review of the case-control studies does not indicate a benefit of prostate cancer screening. An answer has to come from the ERSPC trial, in Europe, and the PLCO trial, in the US, of which the outcomes are expected in 2007-2010.

(C) 2006 European Association of Urology and European Board of Urology. Published by Elsevier B.V. All rights reserved.

* Corresponding author. Department of Public Health, Room AE-136, Erasmus MC, P.O. Box 2040, 3000 CA Rotterdam, The Netherlands. Tel. +31 10 4087446/4638460; Fax: +31 104638474. E-mail address: s.otto@erasmusmc.nl (S.J. Otto).
\end{abstract}

\section{Introduction}

Prostate cancer is the most commonly diagnosed non-cutaneous cancer and the third most common cause of death from cancer in males in the US, estimated at 27,350 deaths in 2006 [1]. The prostate- specific antigen (PSA) test for the early detection of prostate cancer, introduced in late 1980's, has rapidly gained popularity, not only among healthcare providers and their patients, but also among the "healthy" male population. This increasing public awareness and the subsequently rising use of the 
PSA test, as a means of early detection, demands an answer on the question whether early detection of prostate cancer reduces prostate cancer specific mortality.

Currently two randomized controlled trials (RCT) are under way, the European Randomized Study of Screening for Prostate Cancer (ERSPC) [2] and the Prostate Lung Colorectal and Ovary cancer screening trials (PLCO) [3], to study the effectiveness of screening for prostate cancer. The ERSPC was initiated in 1993 and has approximately 205,000 men randomized in eight different centers in Europe. The PLCO trial was also initiated in 1993 and has included approximately 74,000 men, randomized to the prostate arm of the trial. Conditions for a possible common analysis of ERSPC and PLCO trial have been discussed [4].

The ongoing randomized trials will not produce results for several years [5]. Furthermore, the benefit of the RCT design may not be realized if screening in men allocated to the control arm is widespread. This together with the knowledge that prostate cancer has a relatively long natural history, several casecontrol studies were already performed to try speeding up the process of answering the question on the value of prostate cancer screening. Here we review the results of published case-control studies on the efficacy of prostate cancer screening.

\section{Materials and methods}

\subsection{Search strategy}

The PubMed database was searched for papers published until April 2006, in the English language. Search query included the terms prostate cancer (prostate carcinoma) and case-control (case control) in title and/or abstract. For each retrieved paper, the reference lists were checked upon relevant (accessible) literature and the PubMed option Related Articles was consulted.

\subsection{Basic methodological concept of case-control study}

It is well known that the RCT is the study design that provides the most reliable unbiased estimates of the effect of the intervention under study. The RCT is the preferred approach for evaluation of cancer screening programs. In this study design, subjects consenting to participate are randomly allocated to either the screening group, offered screening, or to the control group, that is not offered any screening. The final outcome is assessed according to the intention-to-screen principle where the rate of the cancer-specific mortality is compared between the screening and the control group. In absence of an RCT, e.g. screening test is already widely disseminated [6], information on screening can also be achieved from observational (non-randomized) designs as cohort studies, where groups of individuals is followed up over

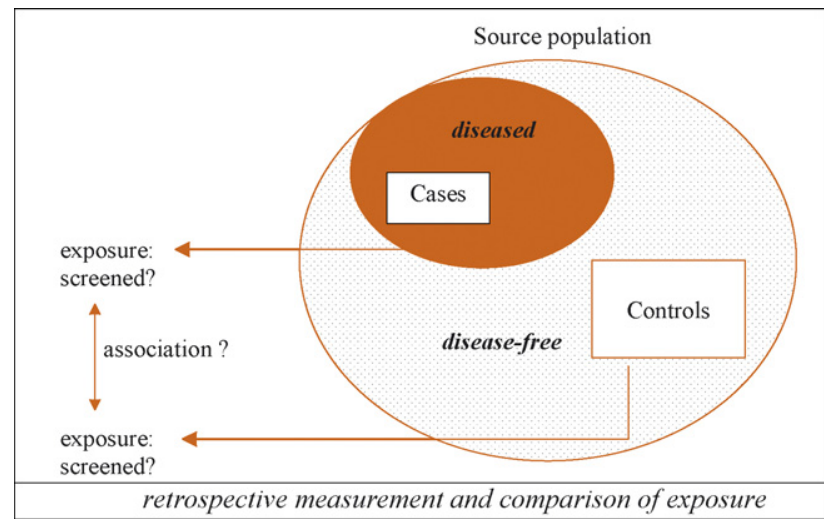

Fig. 1 - Basic concept of the case-control study design.

a period of time, and from time trend analyses, which are based on examination of (aggregated) mortality data of populations across time. Another observational design used to evaluate cancer screening programs is the case-control study. Fig. 1 presents the basic concept of this study design.

In a case-control study of screening, the potential effect of the screening test for prevention of an adverse outcome (e.g. late stage of a disease or mortality) is examined by comparing the exposure to the screening test between those subjects with the adverse outcome, the cases, and those without this outcome, the controls [7]. The evaluation of the efficacy of a test in cancer screening focuses on reducing cancer-specific mortality, hence, the control subject should be alive at the date of death of the case subject and cancer-free at diagnosis of the case subject. As the starting-point of the case-control study is the presence of the adverse outcome in the cases, the exposure to the screening test has to be examined retrospectively, i.e. the screening history of each case and his matched control(s) has to be assessed in the period preceding the diagnosis of the case $[7,8]$. The frequency of screening (ever or never screened) is then compared between the cases and their matched controls which gives an estimate of the strength of the association (Odds ratio, OR), whether screening prevents the adverse outcome (Fig. 1). Importantly, as the screening test is often the same used for diagnostic purposes, true screenings should be distinguished from tests done in relation to symptoms; e.g. both digital rectal examination (DRE) and PSA are used for screening asymptomatic men and for examination of symptomatic subjects.

The cases and controls are selected from the same source population (Fig. 1) and age range within which screening is available. Any difference in screening history between cases and controls would be due to different rates of acceptance of the screening program (or opportunistic screening). Hence, cases and controls may not be appropriately "representative" as might be the case in screening for prostate cancer. It is known that African-Americans have a high-risk for prostate cancer, but the frequency of PSA testing in this population is low compared with the Whites [9].

The key advantages of a case-control study are that it is less costly, less lengthy compared to RCT, does not require a large number of subjects, is appropriate for studying rare conditions 
Table 1 - Description of the case-control studies on prostate cancer screening

\begin{tabular}{|c|c|c|c|c|c|c|c|c|c|c|}
\hline & \multirow[t]{2}{*}{ Source population } & \multirow{2}{*}{$\begin{array}{c}\text { Age at entry } \\
\text { Years }\end{array}$} & \multicolumn{3}{|c|}{ Cases } & \multirow{2}{*}{$\begin{array}{c}\text { Controls } \\
\qquad \mathrm{N}^{\mathrm{a}}\end{array}$} & \multirow[t]{2}{*}{ Index date } & \multicolumn{3}{|c|}{ Screening exposure } \\
\hline & & & $\mathrm{N}$ & Diagnosis PCa & Follow-up endpoint & & & Test(s) & Definition & Window \\
\hline \multicolumn{11}{|l|}{ A. Metastasis } \\
\hline Friedman et al. [19] & health plan & $39-95^{\mathrm{b}}$ & 139 & 1975-1985 & at diagnosis or later & 139 & d & DRE & e & $3 \mathrm{mo}-10 \mathrm{yr}$ \\
\hline Kopec et al [16] & geographic area & $40-84^{c}$ & 236 & $\geq 1$ Jan 1990 & $8 / 1999-5 / 2002$ & 462 & d & PSA & f & e \\
\hline \multicolumn{11}{|l|}{ B. Death } \\
\hline Jacobsen et al. [17] & geographic area & $\mathrm{g}$ & 173 & $<1970-1991$ & 1976-1991 & 346 & d & DRE & e & $10 \mathrm{yr}$ \\
\hline Richert-Boe et al. [18] & health plan & $40-84^{b}$ & 150 & e & 1981-1990 & 299 & d & DRE & f & $10 \mathrm{yr}$ \\
\hline \multirow[t]{2}{*}{ Weinmann et al. [13] } & health plan & $45-84^{c}$ & 171 & e & 1992-1999 & 342 & d & $\mathrm{PSA} / \mathrm{DRE}$ & f & $10 \mathrm{yr}$ \\
\hline & & & & & $1997-1999 / 2000$ & & d & & f & $10 \mathrm{yr}$ \\
\hline Weinmann et al. [14] ${ }^{\mathrm{h}}$ & health plans & $45-84^{c}$ & $608 / 161$ & e & $1997-6 / 2001$ & $608 / 321$ & & PSA/DRE & & \\
\hline Concato et al. $[15]^{\mathrm{i}}$ & medical centers & $\geq 50^{j}$ & 501 & 1991-1995 & 1991-1999 & 501 & d & PSA/DRE & f & $6 \mathrm{mo}-5 \mathrm{yr}$ \\
\hline \multirow{2}{*}{\multicolumn{11}{|c|}{$\begin{array}{l}\text { Abbreviation; PCa, prostate cancer. } \\
\text { a Endpoint free (no metastasis or alive and prostate cancer free at diagnosis of case). }\end{array}$}} \\
\hline & & & & case). & & & & & & \\
\hline \multicolumn{11}{|c|}{ At prostate cancer diagnosis. } \\
\hline \multicolumn{11}{|c|}{${ }^{c}$ At endpoint. } \\
\hline \multicolumn{11}{|c|}{ d Date of diagnosis case. } \\
\hline \multicolumn{11}{|c|}{ e Poorly or not described/defined. } \\
\hline \multirow{2}{*}{\multicolumn{11}{|c|}{$\begin{array}{l}{ }^{f} \text { Well or moderately described/defined. } \\
\text { g Not selection criterion. }\end{array}$}} \\
\hline & & & & & & & & & & \\
\hline \multicolumn{11}{|c|}{ h White, Black men separately. } \\
\hline \multicolumn{11}{|c|}{${ }^{i}$ Nested case control study. } \\
\hline j PCa free as of 1 Jan 1991 & \& ambulatory care $198 \mathrm{~s}$ & & & & & & & & & \\
\hline
\end{tabular}


and diseases with prolonged induction or latency, and it gives the possibility of examining multiple exposures, [8]. However, case-control studies are very susceptible to biases, and the major bias inherent to this study design are misclassification bias and (self-)selection bias. As mentioned above, the screening and diagnostic test are often the same. If during review of medical records, a test performed for diagnostic purposes in a control is erroneously assigned as screening test or a screening test in a case is incorrectly classified as diagnostic test, this ascertainment procedure would introduce misclassification bias which would lead to a bias in favor of screening or against screening, respectively [10]. Subjects attending a screening program or seeking opportunistic screening might differ in the risk of the disease under study, in social-economic status and in access to the screening test. High-risk subjects often decline participation in screening programs. These aspects confer (self-)selection bias, affecting the frequency of screening either in favor or against screening [7].

Although there are several methods described in order to correct for the above-mentioned biases [11,12], the generally regarded optimal design for the evaluation of a populationbased screening program is an RCT. With the RCT design, the study population consists of subjects free of the disease at start of the study, randomly divided into two groups, but perhaps most importantly the investigators assigned the exposure under study. Therefore, an RCT provides the strongest evidence for causality and controls for "unknown" confounders.

\section{Results}

Only seven case-control studies of screening for prostate cancer were identified in the PubMed search, published from 1991 onwards, all conducted in North America. Of these, only three studies assessed the efficacy of either PSA alone or PSA and DRE on prostate cancer mortality [13-15]. One study concerned the effect of PSA screening on the development of metastatic disease [16] and the three remaining examined whether screening by DRE is associated with a reduction in prostate cancer mortality $[17,18]$ or metastatic disease [19]. A description of these studies is presented in Table 1.

The source population from which both cases and controls were selected was composed of medical registries of health-care plans and centers (Kaiser Permanente, [13,14,18,19]; VA Outpatient Clinic System Database, [15,20]) or defined geographical areas (Metropolitan Toronto and surrounding counties, [16], and Olmsted County, Rochester Epidemiology Project, [17]). The subjects were aged 39 years and older at diagnosis of prostate cancer or at reaching study endpoint (metastasis or death from prostate cancer). An exception is the study by Jacobsen et al. [17], where no age range is given, as age was not an inclusion criterion, but prostate cancer death in a defined time period (1976-1991).
Matching of controls to cases was primarily based on age and the date of enrollment in a health plan, a site of ambulatory care in a medical center or an area of residence for the studies using geographical area as source population. The two most recent studies, Weinmann et al. [14] and Concato et al. [15], included the largest number of cases and matched controls (Table 1). The index or reference date was properly defined in all studies, as the first date of suspicion of prostate cancer [8]. In the studies by Weinmann et al., an additional symptoms reference date was selected, defined as first date on which symptoms were noted that were subsequently found to be related to prostate cancer.

The results of the studies examining the potential role of screening on the development of advanced prostate cancer and those on mortality are discussed below separately.

\subsection{Outcome: metastatic prostate cancer}

The results of the two studies with metastasis of the prostate as outcome are presented in Table 2A. The study by Friedman et al. [19] was conducted in men with prostate cancer diagnosed in the pre-PSA era (1979-1985), hence the exposure to DRE screening was identified through review of medical charts. DRE in the three months prior to diagnosis were not included in the analyses to keep the case-control status undisclosed to the chart reviewers. Both cases and matched controls arose from the database of a regional health plan and were within the age group 39-95 years. The race-adjusted odds ratio (OR), up to 10 years before diagnosis, indicated no benefit of screening in prevention of the development of metastatic disease ( $O R=0.9,95 \%$ confidence interval, CI, 0.5-1.7). When DRE performed for evaluation of intestinal or rectal symptoms were included as "screening", the OR became 0.7, but still statistically not significant (95\% CI, 0.4-1.4).

In contrast, Kopec et al. [16] found that PSA screening reduced the risk of metastatic prostate cancer by $35 \%$ (OR $=0.65,95 \% \mathrm{CI}, 0.45-0.93$ ). This beneficial effect was seen in men aged 45-59 years ( $\mathrm{OR}=0.52, \mathrm{CI}, 0.28-0.98)$, and to some extent in those aged 60-84 years (OR $=0.67,95 \% C I, 0.41-1.09)$. Some methodological points of this study should also be noted. The strength of the study is the effort in differentiating between true screening and diagnostic PSA tests, thorough review of medical charts for adjustment of baseline prognostic factors and a sensitivity analysis correcting for positive/suspicious DRE. However, there is a potential nonresponse and information bias in this study as the number of participants returning the mailed 
Table 2 - Adjusted odds ratios of case-control studies on prostate cancer screening with either DRE alone or PSA and DRE

\begin{tabular}{|c|c|c|}
\hline & $\begin{array}{c}\text { PSA +/- DRE } \\
\text { OR (95\% CI) }\end{array}$ & $\begin{array}{l}\text { DRE alone } \\
\text { OR (95\% CI) }\end{array}$ \\
\hline \multicolumn{3}{|c|}{$\begin{array}{l}\text { A. Outcome: metastatic prostate cancer } \\
\text { DRE era }\end{array}$} \\
\hline Friedman et al. [19] & & $\begin{array}{l}0.9(0.5-1.7) \\
0.7(0.4-1.4)^{a}\end{array}$ \\
\hline \multicolumn{3}{|l|}{$\underline{\text { PSA era }}$} \\
\hline Kopec et al. [16] & $\begin{array}{l}0.65(0.45-0.93) \\
0.52(0.28-0.98)^{b} \\
0.67(0.41-1.09)^{c}\end{array}$ & \\
\hline \multicolumn{3}{|c|}{ B. Outcome: prostate cancer mortality } \\
\hline Jacobsen et al. [17] & - & $\begin{array}{l}0.84(0.48-1.46) \\
0.53(0.32-0.90)^{d} \\
0.32(0.20-0.52)^{\mathrm{e}}\end{array}$ \\
\hline \multicolumn{3}{|l|}{$\underline{\text { PSA era }}$} \\
\hline Weinmann et al. [13] & $\begin{array}{l}0.70(0.46-1.1) \\
0.69(0.38-1.2)^{f}\end{array}$ & $0.60(0.38-0.93)$ \\
\hline Weinmann et al. [14] ${ }^{\mathrm{g}}$ & $\begin{array}{l}\mathrm{W}, 0.73(0.55-0.97)^{\mathrm{h}} \\
\mathrm{B}, 1.00(0.59-1.40) \\
1.13(0.63-2.06)\end{array}$ & $\begin{array}{l}\text { W, } 0.65(0.45-0.85)^{\mathrm{h}} \\
\text { B, } 0.86(0.53-1.40)\end{array}$ \\
\hline $\begin{array}{l}\text { Abbreviations: OR, odds ra } \\
\text { a Including DREs for evalu } \\
\text { b Age group 45-69 years. } \\
\text { c Age group 60-84 years. } \\
\text { d Any DRE in exposure wi } \\
\text { e Only DRE in absence of } \\
\text { f Membership > = } 10 \text { years } \\
\text { g Only screening test class } \\
\text { h Study reference date, W }\end{array}$ & & \\
\hline
\end{tabular}

informed consent was low and differed among cases and controls (69\% and 51\%, respectively). The same was true for the availability of medical charts $(93 \%$ and $88 \%$, respectively). This problem is also pointed out in an editorial by Barry [21]. In contrast to the other case-control studies where cases and controls are matched on age and the length of exposure window is kept equal for the case-control pair, the authors of this study matched only on age and considered the difference in exposure window as a confounding factor, which they adjusted for. It may be possible that the adjustment for exposure time has led to significant difference in outcome.

\subsection{Outcome: prostate cancer mortality}

Table 2B shows the corresponding odds ratios of the case-control studies assessing the potential benefit of prostate cancer screening with prostate cancer mortality as endpoint. The two DRE case-control studies, conducted in the pre-PSA era, showed contradictory results, varying from a $68 \%$ significant preventive effect (OR 0.32, 95\% CI 0.20-0.52; [17]) to no effect of DRE screening on prostate cancer mortality (the odds ratio was not significantly different from 1, OR $0.8495 \%$ CI $0.48-1.46$, [18]). In the study by Jacobsen et al. [17], the analyses were restricted by eliminating the first year before the index date to exclude DREs possibly performed for diagnostic purposes. This restriction could have introduced misclassification bias in favor of screening, hence the reason of the significant result of this study.

Conflicting results were found in the PSA/DRE studies as well. In their first study, Weinmann et al. [13] found no statistical significant effect of the combination of PSA and DRE. However, in their subsequent study with a larger population size [14], a significant $27 \%$ reduction was observed, but this concerned the White male cohort only. This effect seemed to be attributable to DRE screening, as analyses including only DRE revealed statistically significant OR of 0.65 (95\% CI 0.45-0.85) in Whites. In Black men, there was no evidence that cases were less likely to have had PSA/DRE screening than the controls, even when DRE alone was considered. Then again the population size was smaller than that for the White cohort (Table 1). If the White and 
Black cohort data were analyzed jointly, most likely the study would not have yielded a statistically significant result.

Concato et al. [15], adjusting for race, showed no effect of PSA/DRE screening in their nested casecontrol study (OR 1.13, 95\% CI 0.71-1.64). Additional analyses including tests performed in men with BPH symptoms or test for differential diagnosis did not alter the outcome.

Connor et al. [22] have shown that the criteria for selecting matched controls to cases, the choice of the time scale of screening and the method to measure exposure of screening in case-control studies can considerably affect the results due to biases introduced. The retrospective identification of screening exposure, particularly for prostate cancer, remains a difficult issue. In all studies reviewed, determination of the reason of the tests were done through review of medical records and in the most recent PSA/DRE studies [14,15], the authors made use of algorithms to classify the PSA and DRE findings as definitely screening, probably screening or diagnostic tests, accounting for $\mathrm{BPH}$ and its symptoms. The study of Jacobsen et al. [17], which assessed whether screening with DRE reduced prostate cancer mortality, did not take examinations that were performed for evaluation of $\mathrm{BPH}$ symptoms into account. Screening was defined as the absence of hematuria, bone pain and pelvic pain at the time of DRE. However, even when the analysis where performed with exposure being any DRE, an adjusted OR of 0.53 (95\% CI $0.32-0.90)$ was observed. The importance of accurate classification of true screening and diagnostic tests in casecontrol studies of the efficacy of prostate cancer screening have been demonstrated by several authors [10,23-25]. If screening tests are not properly defined, the proportion of subjects classified as screened will be distorted leading to biased study results. Hence, the studies of Weinmann et al. [14] and Concato et al. [15] were likely not biased by misclassification of the exposure.

However, the significant outcome for DRE alone in White men in the large study of Weinmann et al. [14] might reflect (self-)selection bias as prostate cancer screening is opportunistic and, as mentioned before, the rate of screening in the US is higher among the White population.

\section{Discussion}

A large number of observational studies on the evidence of prostate cancer screening and mortality reduction have been published with contradictory outcome [26-29]. Coldman et al. [30] observed that high screening intensity is not associated with large reductions in prostate cancer mortality, when compared with geographic areas with low or medium screening intensity. Using incidence-based mortality rates, Chu et al. [31] demonstrated that the decrease in mortality rates among men with distant disease diagnosis accounted for the overall decline in prostate cancer mortality trends in the USA. In the non-randomized prospective Tyrol screening study initiated in 1993, men aged 45-74 years were offered the PSA test [28,32]. Over the period 1996-2000, the trends in prostate cancer mortality showed that the mortality in Tyrol was 35\% lower than in the rest of Austria.

The results of the Quebec RCT, started in 1988, only further contributed to the controversy on prostate cancer screening. In the two publications of this RCT, decreases of 69\% [33] and 62\% [34] in the prostate cancer mortality were reported, when screened men were compared to unscreened men. The proper analyses according to the intention-to screen principle yielded relative risks of 1.06 [33] and 1.08 [34], respectively, indicating no effect of screening, particularly due to lack of power.

From this current review of case-control studies we can conclude that the evidence for a beneficial effect of prostate cancer screening on mortality reduction is still not unambiguous. The major limitation of the case-control studies reviewed is the potential misclassification of the exposure, PSA tests and DREs, particularly in presence of other non-cancer symptoms of the prostate, and the accuracy of the index date. The studies by Concato et al. [15] and Weinmann et al. [14] seem not to be weighed down by these aspects, but there is evidence of selection bias in Weinmann's study as a significant result was only seen in White men.

Decisions on population-based screening require solid evidence of its benefits on the reduction of cancer-specific mortality. The costs and the effects of quality of life will be important additional outcomes. It is clear from the studies discussed above that observational study designs were not able to provide the evidence needed for this important decision. Level one evidence, i.e. from a prospective RCT is definitely still required. Waiting for the outcome of the two large-scaled randomized trials $[3,35]$ seems the only rational approach. However, the question remains how a result of an intention-to-screen analysis from an RCT of screening (effectiveness estimate) can be translated to daily practice, since there is always a certain level of non-compliance in the screening arm and 
contamination in the control arm. To answer the question of the value of early detection on an individual level (efficacy estimate) a secondary analysis with both correction for non-compliance and contamination is needed [36]. This type of analysis determines the magnitude of benefit in terms of prostate cancer risk/mortality reduction by attending a screening program.

When non-compliance and contamination are large in a RCT, investigators might make comparisons of those who actually received the intervention with those who did not, similar as in the publication of the Quebec trial $[33,34]$. This would lead to large effect estimates, but is not valid, as the randomization status is not complied with, introducing selection bias in the analyses. A method to adjust for non-compliance and contamination in an RCT has been developed by Cuzick et al. [36], which in the Rotterdam section of ERSPC, will be applied in addition to the main intention-to-screen analysis, to give an effect estimate after adjustment for noncompliance and contamination. A case-control study using the ERSPC data will also be conducted afterwards to give an impression of the magnitude of all known biases inherent to the case-control study on the outcome of a population-based trial.

\section{Conclusion}

In conclusion, as for other observational studies, the current available data from case-control studies on the value of early detection of prostate cancer in reducing disease specific mortality yielded inconclusive evidence. Hence, the presumably most debated topic in urologic practice remains uncertain at this time point. An answer has to come from wellconducted RCTs, the ERSPC in Europe and the PLCO trial in the US, of which the outcomes can be expected in 2007-2010.

With the growing awareness of prostate cancer screening not only among physicians, but also among the general population, a point of concern for these RTCs can be the generalizibility to population level. As described above valid statistical techniques are available to correct for this issue to obtain a reliable estimate of the true effect of undergoing the screening test. Until then, it is advisable that men who wish to be screened should be well informed of both the benefits and possible harms (i.e. overdiagnosis, overtreatment, consequences of aggressive treatment as sexual, urinary and bowel dysfunctions) of prostate cancer screening, realizing that the major benefit on prostate cancer mortality reduction has not been shown yet.

\section{References}

[1] Jemal A, Siegel R, Ward E, Murray T, Xu J, Smigal C, et al. Cancer statistics, 2006. CA Cancer J Clin 2006;56: 106-30.

[2] Roobol MJ, Schröder FH, editors. The European Randomized Study of Screening for Prostate Cancer (ERSPC): rationale, structure and preliminary results 1994-2003. BJU Int Suppl 2003;92:1-117.

[3] Prorok PC, Andriole GL, Bresalier RS, Buys SS, Chia D, Crawford ED, et al. Design of the Prostate, Lung, Colorectal and Ovarian (PLCO) Cancer Screening Trial. Control Clin Trials 2000;21:273S-309S.

[4] Auvinen A, Rietbergen JB, Denis LJ, Schroder FH, Prorok PC. Prospective evaluation plan for randomised trials of prostate cancer screening. The International Prostate Cancer Screening Trial Evaluation Group. J Med Screen 1996;3:97-104.

[5] de Koning HJ, Liem MK, Baan CA, Boer R, Schröder FH, Alexander FE, et al. Prostate cancer mortality reduction by screening: Power and time frame with complete enrollment in the European randomised screening for prostate cancer (ERSPC) trial. Int J Cancer 2002;98: 268-73.

[6] Laara E, Day NE, Hakama M. Trends in mortality from cervical cancer in the Nordic countries: association with organised screening programmes. Lancet 1987;1:1247-9.

[7] Weiss NS. Application of the case-control method in the evaluation of screening. Epidemiol Rev 1994;16:102-8.

[8] Cronin KA, Weed DL, Connor RJ, Prorok PC. Case-control studies of cancer screening: theory and practice. J Natl Cancer Inst 1998;90:498-504.

[9] Etzioni R, Berry KM, Legler JM, Shaw P. Prostate-specific antigen testing in black and white men: an analysis of medicare claims from 1991-1998. Urology 2002;59: 251-5.

[10] Concato J. What is a screening test? Misclassification bias in observational studies of screening for cancer. J Gen Intern Med 1997;12:607-12.

[11] Duffy SW, Cuzick J, Tabar L, Vitak B, Chen THH, Yen MF, et al. Correcting for Non-Compliance Bias in Case-Control Studies to Evaluate Cancer Screening Programmes. Appl Statist 2002;51:235-43.

[12] Weiss NS, Dhillon PK, Etzioni R. Case-control studies of the efficacy of cancer screening: overcoming bias from nonrandom patterns of screening. Epidemiology 2004; 15:409-13.

[13] Weinmann S, Richert-Boe K, Glass AG, Weiss NS. Prostate cancer screening and mortality: a case-control study (United States). Cancer Causes Control 2004;15: 133-8.

[14] Weinmann S, Richert-Boe KE, Van Den Eeden SK, Enger SM, Rybicki BA, Shapiro JA, et al. Screening by prostatespecific antigen and digital rectal examination in relation to prostate cancer mortality: a case-control study. Epidemiology 2005;16:367-76.

[15] Concato J, Wells CK, Horwitz RI, Penson D, Fincke G, Berlowitz DR, et al. The effectiveness of screening for prostate cancer: a nested case-control study. Arch Intern Med 2006;166:38-43. 
[16] Kopec JA, Goel V, Bunting PS, Neuman J, Sayre EC, Warde $\mathrm{P}$, et al. Screening with prostate specific antigen and metastatic prostate cancer risk: a population based case-control study. J Urol 2005;174:495-9, discussion 499.

[17] Jacobsen SJ, Bergstralh EJ, Katusic SK, Guess HA, Darby $\mathrm{CH}$, Silverstein $\mathrm{MD}$, et al. Screening digital rectal examination and prostate cancer mortality: a population-based case-control study. Urology 1998;52:173-9.

[18] Richert-Boe KE, Humphrey LL, Glass AG, Weiss NS. Screening digital rectal examination and prostate cancer mortality: a case-control study. J Med Screen 1998;5: 99-103.

[19] Friedman GD, Hiatt RA, Quesenberry Jr CP, Selby JV. Casecontrol study of screening for prostatic cancer by digital rectal examinations. Lancet 1991;337:1526-9.

[20] Concato J, Peduzzi P, Kamina A, Horwitz RI. A nested case-control study of the effectiveness of screening for prostate cancer: research design. J Clin Epidemiol 2001; 54:558-64.

[21] Barry MJ. The PSA Conundrum. Arch Intern Med 2006; 166:7-8.

[22] Connor RJ, Boer R, Prorok PC, Weed DL. Investigation of design and bias issues in case-control studies of cancer screening using microsimulation. Am J Epidemiol 2000;151:991-8.

[23] Gill TM, Horwitz RI. Evaluating the efficacy of cancer screening: clinical distinctions and case-control studies. J Clin Epidemiol 1995;48:281-92.

[24] Godley PA, Rathore SS, Kshirsagar AV, Amamoo MA, Schell MJ, Freeman J, et al. Retrospective classification of prostate-specific antigen tests: differentiating screening from diagnostic clinical encounters. J Clin Epidemiol 2001;54:884-8.

[25] Hoffman RM, Adams-Cameron ML, Murata GH. Misclassifying the indications for prostate-specific antigen testing may bias case-control studies of the efficacy of prostate cancer screening. J Clin Epidemiol 2004;57: 1071-5.

[26] Oliver SE, Gunnell D, Donovan JL. Comparison of trends in prostate-cancer mortality in England and Wales and the USA. Lancet 2000;355:1788-9.

\section{CME questions}

Please visit www.eu-acme.org/europeanurology to answer these EU-ACME questions on-line. The EU-ACME credits will then be attributed automatically.

1. The major drawback of the case control study design is that:

A. It is not suitable for rare conditions.

B. The retrospective design induces susceptibility for selection and misclassification bias.

C. The intervention under study (cause) is not applied by the investigator.

D. It is logistically inefficient.
[27] Oliver SE, May MT, Gunnell D. International trends in prostate-cancer mortality in the "PSA ERA". Int J Cancer 2001;92:893-8.

[28] Bartsch G, Horninger W, Klocker H, Reissigl A, Oberaigner W, Schonitzer D, et al. Prostate cancer mortality after introduction of prostate-specific antigen mass screening in the federal state of Tyrol, Austria. Urology 2001;58: 417-24.

[29] Lu-Yao G, Albertsen PC, Stanford JL, Stukel TA, WalkerCorkery ES, Barry MJ. Natural experiment examining impact of aggressive screening and treatment on prostate cancer mortality in two fixed cohorts from Seattle area and Connecticut. BMJ 2002;325:740.

[30] Coldman AJ, Phillips N, Pickles TA. Trends in prostate cancer incidence and mortality: an analysis of mortality change by screening intensity. CMAJ 2003;168:31-5.

[31] Chu KC, Tarone RE, Freeman HP. Trends in prostate cancer mortality among black men and white men in the United States. Cancer 2003;97:1507-16.

[32] Horninger W, Berger A, Pelzer A, Klocker H, Oberaigner W, Schonitzer D, et al. Screening for prostate cancer: updated experience from the Tyrol study. Can J Urol 2005;12:7-13, discussion 92-3.

[33] Labrie F, Candas B, Dupont A, Cusan L, Gomez JL, Suburu $\mathrm{RE}$, et al. Screening decreases prostate cancer death: first analysis of the 1988 Quebec prospective randomized controlled trial. Prostate 1999;38:83-91.

[34] Labrie F, Candas B, Cusan L, Gomez JL, Bélanger A, Brousseau G, et al. Screening decreases prostate cancer mortality: 11-year follow-up of the 1988 Quebec prospective randomized controlled trial. Prostate 2004;59: 311-8.

[35] de Koning HJ, Auvinen A, Berenguer Sanchez A, Calais Da Silva F, Ciatto S, Denis L, et al. Large-scale randomized prostate cancer screening trials: Program performances in the European randomized screening for prostate cancer trial and the prostate, lung, colorectal and ovary cancer trial. Int J Cancer 2002;97:237-44.

[36] Cuzick J, Edwards R, Segnan N. Adjusting for non-compliance and contamination in randomized clinical trials. Stat Med 1997;16:1017-29.

2. The strongest evidence on the effect of an intervention on disease outcome is a RCT since

A. It has a prospective study design.

B. It is based on individual data.

C. It has an observational approach.

D. The exposure under study is applied by the investigator.

3. The term 'index or reference date' refers to the:
A. date of diagnosis of the case
B. date of death of the case
C. date on which the case was screened
D. date on which the control was screened 
4. Which of the following statements is correct in a case-control study with prostate cancer death as endpoint?

A. A case is a subject who was diagnosed with prostate cancer and died from lung cancer

B. A control is a subject who was diagnosed with prostate cancer before his matched case and died from prostate cancer

C. A case is a subject who was diagnosed with prostate cancer and still alive

D. A control is a subject without prostate cancer before the date of diagnosis of his matched case and still alive at death of this case

5. Regarding the effect of screening on prostate cancer mortality following statement is correct:

A. Case-control studies demonstrated the effectivity of PSA screening
B. The Quebec RCT showed conclusive and unbiased outcome

C. Trend analyses are useful in analyzing the effect of screening

D. The evidence of prostate cancer screening and mortality has yet to come from the RCTs

6. The outcome of a RCT on the effectiveness of a screening program can

A. automatically be translated to daily practice.

B. be interpreted as an efficacy estimate of the screening program applied.

C. give an efficacy estimate after correction for both non-compliance and contamination.

D. give a reliable estimate on the effectiveness of the program when using an intention-to-treat analysis. 